\title{
Couplonics Of Cyclic Ternary Systems: From Coupled Periodic Waveguides To Discrete Photonic Crystals
}

\author{
Yann G. Boucher ${ }^{1,2}$ \\ ${ }^{1}$ Université Européenne de Bretagne, ENIB, France \\ ${ }^{2}$ CNRS, UMR 6082 FOTON, ENSSAT, 6 rue de Kerampont, BP 80518, F-22305 Lannion, France \\ E-mail: boucherdenib.fr
}

\begin{abstract}
In the context of coupled periodic waveguides, "couplonics" refers to the rigorous equivalence between continuous wave coupling and localized interactions. We extend it here to a cyclic ternary system, looked upon as the simplest discrete photonic crystal with actual periodic boundary conditions. A linear decomposition on a supermode basis enables one to reduce the original six-wave problem to three independent two-wave distributed Bragg reflectors (or 1D PC).
\end{abstract}

\section{Introduction}

Whatever the frequency range, a system made of coupled periodic waveguides (CPW) ensures simultaneously filtering and addressing functions [1-2]. When dealing with CPW, "couplonics" [3-4] stems from the formal identification between the continuous configuration, represented by an invariant evolution operator, and the discrete one, seen as multiple-port networks interconnected by segments of transmission lines [5]. In a symmetric system limited to only two CPW, a linear decomposition on the even/odd eigenbasis (that of the "supermodes") enables one to express any scattering parameter as a linear superposition of S-parameters of the underlying even/odd two-port networks. On the other hand, for each supermode, the system can be thought of as an instance of Distributed Bragg Reflector (DBR), well described in terms of Coupled-Mode Theory (CMT) [6].

The ternary configuration is a bit more complicated. Even if the periodic waveguides are identical, the symmetries of the system depend on the distribution of the mutual coupling. In what follows, we consider first an ideal system made of three identical single-mode $\Lambda$-periodic waveguides, of average propagation constant $\beta$ along the $z$-axis, symmetrically coupled in a cyclic way [Fig. 1]. Such a configuration could be achieved, for instance, in a three-core optical fibre with a photo-induced index grating. For the sake of clarity, the unit cell of each waveguide is assumed symmetrical and lossless. Time dependence is taken as $\exp (+i \omega t)$. Physically speaking, co-directional coupling is related to the mutual overlap of the guided modes through their evanescent part, whereas contra-directional coupling comes from the periodic modulation of the effective index along the $z$-axis.
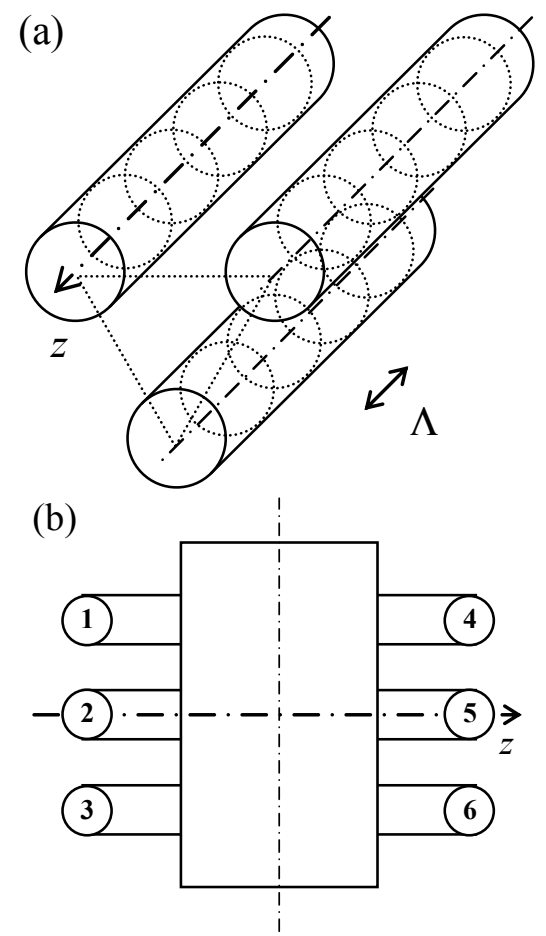

Figure 1: Cyclic ternary system made of single-mode $\Lambda$ periodic waveguides: (a) schematic representation; (b) unit cell seen as a symmetrical six-port network.

\section{Evolution operator}

\subsection{Supermodes of the non-periodic structure}

In the usual perturbative approach [7], when only codirectional coupling occurs, with coupling constant $\chi$ (real and positive without loss of generality), slowly varying envelopes $A_{n}$ of fields $F_{n}=A_{n} \exp (-i \beta z)$ should obey:

$$
i \frac{\partial}{\partial z}\left(\begin{array}{l}
A_{1} \\
A_{2} \\
A_{3}
\end{array}\right)=[\mathrm{K}]\left(\begin{array}{l}
A_{1} \\
A_{2} \\
A_{3}
\end{array}\right)=\left(\begin{array}{lll}
0 & \chi & \chi \\
\chi & 0 & \chi \\
\chi & \chi & 0
\end{array}\right)\left(\begin{array}{l}
A_{1} \\
A_{2} \\
A_{3}
\end{array}\right),
$$

Note that the value of $\beta$ itself takes also into account the influence of the neighbouring waveguides.

Eigenvalues of operator $[\mathrm{K}]$ are $\lambda_{a}=+2 \chi, \lambda_{b}=\lambda_{c}=-\chi$. We 
establish an orthogonal eigenmode basis such as:

$$
\begin{aligned}
& \left(\begin{array}{l}
A_{1} \\
A_{2} \\
A_{3}
\end{array}\right)=[\mathrm{P}]\left(\begin{array}{l}
A_{a} \\
A_{b} \\
A_{c}
\end{array}\right), \\
& {[\mathrm{P}]=\frac{1}{\sqrt{6}}\left(\begin{array}{ccc}
\sqrt{2} & -\sqrt{3} & 1 \\
\sqrt{2} & 0 & -2 \\
\sqrt{2} & \sqrt{3} & 1
\end{array}\right) .}
\end{aligned}
$$

In that basis, the evolution operator is obviously diagonal. Note that in this cyclic case, the degeneracy is not completely lifted $\left(\lambda_{b}=\lambda_{c}\right)$.

Matrix $[\mathrm{P}]$ is unitary. The inverse matrix reads:

$$
[\mathrm{P}]^{-1}=\frac{1}{\sqrt{6}}\left(\begin{array}{ccc}
\sqrt{2} & \sqrt{2} & \sqrt{2} \\
-\sqrt{3} & 0 & \sqrt{3} \\
1 & -2 & 1
\end{array}\right) .
$$

\subsection{Cyclic ternary periodic structure}

The $(6 \times 6)$ evolution operator $[\mathrm{K}]$ connecting the envelopes $\left(C_{n}^{+}, C_{n}^{-}\right)$of co- and contra-propagating fields ${F_{n}}^{+}=C_{n}^{+} \exp \left(-i \beta_{B} z\right)$ and $F_{n}{ }^{-}=C_{n}{ }^{-} \exp \left(+i \beta_{B} z\right)$ reads:

$$
i \frac{\partial}{\partial z}\left(\begin{array}{l}
C_{1}^{+} \\
C_{1}^{-} \\
C_{2}^{+} \\
C_{2}^{-} \\
C_{3}^{+} \\
C_{3}^{-}
\end{array}\right)=[\mathrm{K}]\left(\begin{array}{l}
C_{1}^{+} \\
C_{1}^{-} \\
C_{2}^{+} \\
C_{2}^{-} \\
C_{3}^{+} \\
C_{3}^{-}
\end{array}\right)
$$

with

$$
[\mathrm{K}]=\left(\begin{array}{cccccc}
\delta & \kappa & \chi & \xi & \chi & \xi \\
-\kappa & -\delta & -\xi & -\chi & -\xi & -\chi \\
\chi & \xi & \delta & \kappa & \chi & \xi \\
-\xi & -\chi & -\kappa & -\delta & -\xi & -\chi \\
\chi & \xi & \chi & \xi & \delta & \kappa \\
-\xi & -\chi & -\xi & -\chi & -\kappa & -\delta
\end{array}\right)
$$

where $\beta_{B}=\pi / \Lambda$ denotes the Bragg wavevector, $\delta=\beta-\beta_{B}$ the detuning, $\kappa$ and $\xi$ the (real positive) constants for direct and crossed contra-directional coupling. The latter one is required for the sake of completeness.

A straightforward calculation shows that, in the eigenmode basis:

$$
i \frac{\partial}{\partial z}\left(\begin{array}{l}
C_{x}^{+} \\
C_{x}^{-}
\end{array}\right)=\left(\begin{array}{cc}
\delta_{x} & \kappa_{x} \\
-\kappa_{x} & -\delta_{x}
\end{array}\right)\left(\begin{array}{l}
C_{x}^{+} \\
C_{x}^{-}
\end{array}\right),
$$

for $x \in\{a, b, c\}$, with

$$
\begin{array}{ll}
\delta_{a}=\delta+2 \chi, & \delta_{b}=\delta_{c}=\delta-\chi, \\
\kappa_{a}=\kappa+2 \xi, & \kappa_{b}=\kappa_{c}=\kappa-\xi .
\end{array}
$$

We recognize the typical equation for contra-directional mode coupling in a Distributed Bragg Reflector (DBR), each "eigen-DBR" being characterized by a forbidden band of bandwidth $2 \kappa_{x}$, centred on $\delta_{x}=0$ [7]. This result can be thought of as a partial lift of degeneracy. Taken separately, each waveguide is characterised by a forbidden band of width $2 \kappa$ centred on $\delta=0$. In terms of supermodes, coupling constant $\chi$ is responsible for a shift of the band centres, whereas coupling constant $\xi$ affects both the rejection rate and the bandwidth.

The transfer matrix $\left[\mathrm{m}_{x}\right]$ for a unit cell is such as:

$$
\left(\begin{array}{l}
C_{x}^{+}(0) \\
C_{x}^{-}(0)
\end{array}\right)=\left[\mathrm{m}_{x}\right]\left(\begin{array}{l}
C_{x}^{+}(\Lambda) \\
C_{x}^{-}(\Lambda)
\end{array}\right) .
$$

With $\gamma_{x}=\left[\left|\kappa_{x}\right|^{2}-\delta_{x}^{2}\right]^{1 / 2}$, its elements are:

$$
\begin{aligned}
& m_{x 11}=-\left[\cosh \left(\gamma_{x} \Lambda\right)+i\left(\frac{\delta_{x} \Lambda}{\gamma_{x} \Lambda}\right) \sinh \left(\gamma_{x}\right)\right], \\
& m_{x 12}=i\left(\frac{\kappa_{x} \Lambda}{\gamma_{x} \Lambda}\right) \sinh \left(\gamma_{x} \Lambda\right)=m_{x 21}, \\
& m_{x 22}=-\left[\cosh \left(\gamma_{x} \Lambda\right)-i\left(\frac{\delta_{x} \Lambda}{\gamma_{x} \Lambda}\right) \sinh \left(\gamma_{x} \Lambda\right)\right] .
\end{aligned}
$$

For a structure made of $N$ unit cells, the reflectance and transmittance read:

$$
\begin{aligned}
& r_{x}=\frac{M_{x 21}}{M_{x 11}}=\frac{-i \kappa_{x} \sinh \left(\gamma_{x} L\right)}{\gamma_{x} \cosh \left(\gamma_{x} L\right)+i \delta_{x} \sinh \left(\gamma_{x} L\right)}, \\
& t_{x}=\frac{(-1)^{N} \gamma_{x}}{\gamma_{x} \cosh \left(\gamma_{x} L\right)+i \delta_{x} \sinh \left(\gamma_{x} L\right)},
\end{aligned}
$$

with $L=N \Lambda$.

\subsection{Scattering parameters}

The system is therefore totally determined by four coefficients only:

$$
\begin{array}{ll}
r_{a}, & r_{b}=r_{c}, \\
t_{a}, & t_{b}=t_{c} .
\end{array}
$$

It is not difficult to establish that:

$$
\begin{aligned}
& S_{11}=S_{22}=S_{33}=\left(r_{a}+2 r_{b}\right) / 3=r_{/ /}, \\
& S_{41}=S_{52}=S_{63}=\left(t_{a}+2 t_{b}\right) / 3=t_{/ /}, \\
& S_{21}=S_{32}=S_{13}=\left(r_{a}-r_{b}\right) / 3=r_{\iota}, \\
& S_{51}=S_{62}=S_{43}=\left(t_{a}-t_{b}\right) / 3=t_{\iota} .
\end{aligned}
$$

The system is both symmetrical and reciprocal: $\forall(p, q)$, $S_{p q}=S_{q p}$. The $S$-parameters can take only one out of 4 values: direct transmission $t_{/ /}\left(S_{41}\right.$ and the like), direct reflection $r_{/ /}\left(S_{p p}\right)$, crossed transmission $t_{\perp}\left(S_{51}\right.$ and the like $)$, crossed reflection $r_{\perp}\left(S_{21}\right.$ and the like). 


\section{Discrete configuration}

\subsection{Transmittance and reflectance}

Let us now consider the discrete configuration. The whole system is totally determined by four coefficients only: $\left(r_{/ /}\right.$, $\left.r_{\perp}, t_{/ /}, t_{\perp}\right)$. Symmetries being the same as in the continuous case, eigenmodes of the former are also eigenmodes of the latter. We get:

$$
\begin{array}{ll}
r_{a}=r_{/ /}+2 r_{\llcorner}, & r_{b}=r_{c}=r_{/ /}-r_{\llcorner}, \\
t_{a}=t_{/ /}+2 t_{\llcorner}, & t_{b}=t_{c}=t_{/ /}-t_{\llcorner} .
\end{array}
$$

Once again, the six-port network can be decomposed as a linear superposition of two-port networks.

\subsection{Couplonic identification}

For any eigenmode, the corresponding two-port network can always be expressed in terms of coupled-mode theory. This stems from the symmetry properties of the unitary transfer matrix of one unit cell, as elegantly established in 1997 by Matuschek et al. in the case of an arbitrary multilayer Distributed Bragg Reflector [8]. For a long time, it has been believed that CMT holds only in a perturbative way (for small index modulations), and only if the interaction length $L$ is much greater than period $\Lambda$, but we would like to emphasise that, as shown in [8], the equivalence remains mathematically exact at the scale of $\Lambda$ only, whatever the precise content of the unit cell.

This enables one to define without ambiguity, for any eigenmode $x \in\{a, b, c\}$, an equivalent coupling constant $\kappa_{x}$ and an equivalent detuning $\delta_{x}$.

Or, to be more specific, since we work at the scale of one unit cell, we can establish dimensionless parameters $\left(\kappa_{a} \Lambda, \delta_{a} \Lambda\right)$ and $\left(\kappa_{b} \Lambda, \delta_{b} \Lambda\right)$, which in turn lead to four dimensionless parameters $(\delta \Lambda, \kappa \Lambda, \chi \Lambda, \xi \Lambda)$ that completely describe the whole system:

$$
\begin{gathered}
\delta \Lambda=\frac{\delta_{a} \Lambda+\lambda \delta_{b}}{3}, \\
\chi \Lambda=\frac{\delta_{a} \Lambda-\delta_{b} \Lambda}{3}, \\
\kappa \Lambda=\frac{\kappa_{a} \Lambda+\lambda \kappa_{b}}{3}, \\
\xi \Lambda=\frac{\kappa_{a} \Lambda-\kappa_{b} \Lambda}{3} .
\end{gathered}
$$

We call couplons these parameters, which should be interpreted as elementary quanta of detuning or coupling that take place at the scale of one unit cell. As a matter of fact, as can be seen from Eqns.(5-6), the responses of the system made of $N$ cells involves only multiple quantities such as $\delta L=N \delta \Lambda, \kappa L=N \kappa \Lambda, \chi L=N \chi \Lambda, \xi L=N \xi \Lambda$.

\subsection{Normalised spectral responses}

Whatever the configuration - continuous or discrete -, the spectral responses take the same form. For instance, we draw in Figure 2 the spectral transmittance of a cyclic ternary CPW system for $\kappa L=2, \chi L=1, \xi L=0.25$, as compared to the spectral response of a single (uncoupled) periodic waveguide. The coupling is obviously responsible for a partial lift of degeneracy: taken separately, each periodic waveguide is characterised by a forbidden band centred on $\delta L=0$, of bandwidth $2|\kappa L|$. In terms of supermodes, codirectional coupling constant $\chi$ is responsible for a shift of the stop-band, whereas crossed contradirectional coupling constant $\xi$ modifies both its rejection rate and bandwidth.

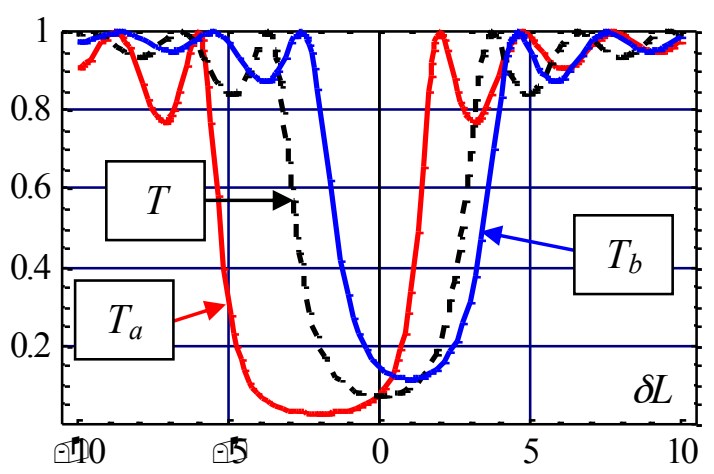

Figure 2: Normalised spectral transmittance $T_{a}$ and $T_{b}$ of the supermodes $\left(T_{x}=\left|t_{x}\right|^{2}\right)$, as compared to transmittance $T$ of a single (uncoupled) periodic waveguide $(\kappa L=2$, $\chi L=1, \xi L=0.25$ )

As a matter of fact, the maximum reflectance and minimum transmittance are given by [7]:

$$
\begin{aligned}
& R_{x \max }=\tanh ^{2}\left(\left|\kappa_{x} L\right|\right), \\
& T_{x \min }=1-\tanh ^{2}\left(\left|\kappa_{x} L\right|\right) .
\end{aligned}
$$

The reflectance is reported in Figure 3 for the same set of reduced parameters:

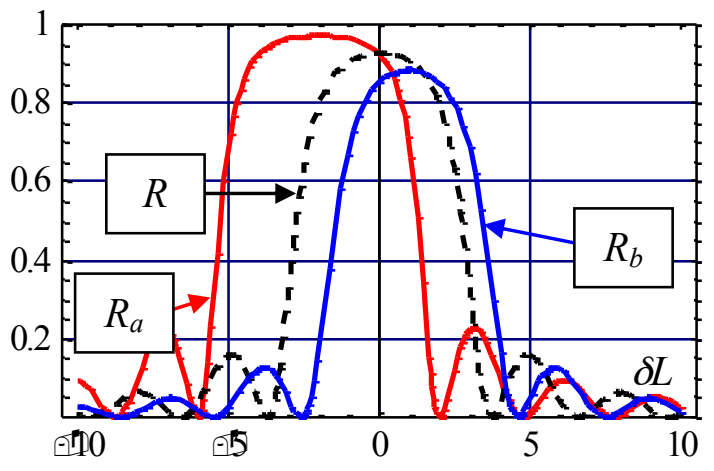

Figure 3: Normalised spectral reflectance $R_{a}$ and $R_{b}$ of the supermodes $\left(R_{x}=\left|r_{x}\right|^{2}\right)$, as compared to transmittance $R$ of a single (uncoupled) periodic waveguide $(\kappa L=2, \chi L=1, \xi L=0.25)$ 


\subsection{Universal Bragg reflector}

The spectral response of a standard DBR is the key to that of the whole system, whatever the precise values of the "couplonic" parameters. Moreover, any actual lossless DBR can be reduced to one instance of a "universal" lossless Bragg reflector, as schematically depicted in Figure 4. As a matter of fact, only two parameters $(\kappa L, \delta L)$ govern its behaviour.

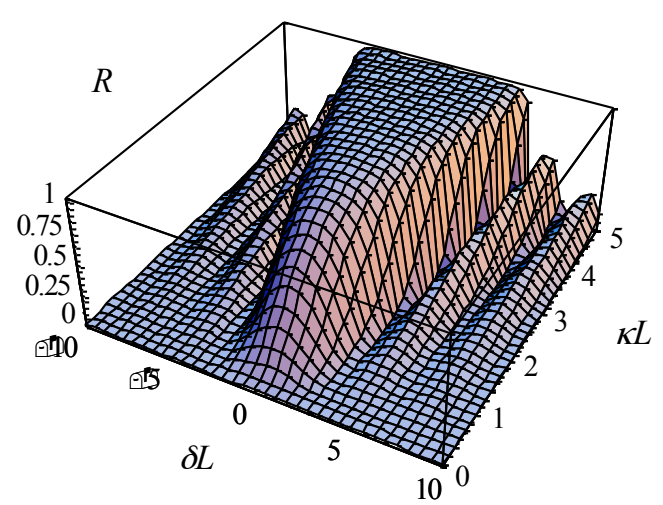

Figure 4: Normalised reflectance $R=\left|M_{21} / M_{11}\right|^{2}$ of a "universal" DBR.

Each spectrum of Figure 3 can be recovered by following a specific path over the universal relief of Figure 4. By an obvious topographic analogy [9], we can speak of "couplonic alpinism".

\section{Conclusions}

We have established analytically, in terms of reduced dimensionless parameters, the spectral responses of a cyclic ternary system made of symmetrically coupled periodic waveguides. In the supermode basis, the method stems from a rigorous mathematical identification between the continuous and discrete configurations.

As paradoxical as that may seem, any discrete ternary systems with the right symmetries can be described, without any approximation, by a continuous evolution operator: Initially looked upon as resulting from a mere approximation, couplonic parameters $(\kappa \Lambda, \delta \Lambda, \chi \Lambda, \xi \Lambda)$ prove much more rigorous than expected. The usual distinction between localised and distributed interactions is therefore blurred.

Taking losses into account would not cause any special difficulty: it would be enough to add two new reduced parameters, corresponding respectively to average losses and to loss-modulation (loss coupling). Optical amplification would appear just as straightforward, the structure becoming a cyclic array of coupled Distributed Feedback (DFB) emitters [10].

The so-called "couplonic" approach is an elegant as well as powerful theoretical tool, not only for studying spectrally selective splitters, but also for the analysis or synthesis of discrete electromagnetic crystals of finite size [5]. Moreover, it comes well within the framework of current research on discrete photonics based on coupled waveguides [11-12].

\section{Acknowledgements}

The author would like to thank Nadia Belabas and her coworkers from Laboratoire de Photonique et de Nanostructures (LPN, CNRS UPR 20), Marcoussis, France, for inspiring discussions.

\section{References}

[1] S. Boscolo, M. Midrio, C.G. Someda, Coupling and Decoupling of Electromagnetic Waves in Parallel 2-D Photonic Crystal Waveguides, IEEE J. Quantum Electron., Vol. 38 (1), 47-53, 2002.

[2] J. Zimmermann, M. Kamp, A. Forchel, R. März, Photonic crystal waveguide directional couplers as wavelength selective optical filters, Optics Comm., Vol. 230, 387-392, 2004.

[3] Y.G. Boucher, Fundamentals of Couplonics, Proc. SPIE Photonics Europe, Strasbourg, France, Vol. 6182, $61821 \mathrm{E}, 2006$.

[4] Y.G. Boucher, A.V. Lavrinenko, D.N. Chigrin, Out-ofphase Coupled Periodic Waveguides: a "couplonic" approach, Optical Quantum Electron., Vol. 39, No. 10-11, 837-847, 2007.

[5] L. Le Floc'h, V. Quintard, J.-F. Favennec, Y. Boucher, Spectral Properties of a Periodic $\mathrm{N} \times \mathrm{N}$ Network of Interconnected Transmission Lines, Microwave Optical Technol. Lett., Vol. 37 (4), 255-259, 2003.

[6] A.A. Barybin and V.A. Dmitriev, Modern Electrodynamics and Coupled-Mode Theory: Application to Guided-Wave Optics, Rinton Press, 2002.

[7] A. Yariv and P. Yeh, Optical Waves in Crystals, Wiley, New York, 1984.

[8] N. Matuschek, F.X. Kärtner, U.Keller, Exact CoupledMode Theories for Multilayer Interference Coatings with Arbitrary Strong Index Modulations, IEEE J. Quantum Electron., Vol. 33 (3), 295-302, 1997.

[9] Y.G. Boucher, L. Le Floc'h, V. Quintard, J.-F. Favennec, "Canonical alpinism" and "canonical surf-riding": a universal tool for normalised parametric analysis of one-dimensional periodic structures, Optical and Quantum Electronics, Vol. 38 (1-3), 203-207, 2006.

[10]H. Kogelnik, C.V. Shank, Coupled-Wave Theory of Distributed Feedback Lasers, J. Appl. Phys., Vol. 43, 2327-2335, 1972.

[11] N. Belabas, S. Bouchoule, I. Sagnes, J.A. Levenson, C. Minot, J.-M. Moison, Confining light flow in weakly coupled waveguide arrays by structuring the coupling constant: towards discrete diffractive optics, Opt. Expr., Vol. 17 (5), 3148-3156, 2009.

[12] E. Feigenbaum, H.A. Atwater, Resonant Guided Wave Networks, Phys. Rev. Lett. Vol. 104, 147402, 2010. 